\title{
THE COMPLETENESS THEOREM FOR ROSSBY NORMAL MODES OF A STABLY STRATIFIED FLAT OCEAN WITH AN ARBITRARY FORM OF SIDE BOUNDARY
}

\author{
BY \\ AKIRA MASUDA \\ University of Tokyo, Tokyo, Japan
}

\begin{abstract}
A mid-latitude flat ocean on a $\beta$-plane has characteristic oscillations called Rossby normal modes, where the motion is governed by the quasigeostrophic vorticity equation. Although the relevant eigenvalue problem differs from the usual one of Hilbert-Schmidt type, a variational proof is obtained that the Rossby normal modes constitute a complete orthonormal set for a basin with an arbitrary profile of stable density stratification and an arbitrary form of side boundary. In particular, for each fixed vertical mode, the set of the horizontal modes is complete and orthonormal in a two-dimensional Hilbert space. General solutions are expressed in terms of Rossby normal modes, not only to the initial-value problem, but also to the response problem of the closed basin.
\end{abstract}

1. Introduction. On account of the unusual reflection of Rossby waves on nonzonal boundaries (LeBlond and Mysak [10]), it has never been easy to understand and predict the evolution or the response of a closed ocean even in linear cases (e.g., Lighthill [11]; Anderson and Gill [1]). The primary purpose of this paper is to provide a general linear solution to the initial-value problem and the response problem of a closed ocean at mid-latitudes through a study of the set of characteristic oscillations of a closed basin on a $\beta$-plane.

Those oscillations, known as Rossby normal modes, can be calculated rather easily together with the associated characteristic frequencies (e.g., Longuet-Higgins [12]; Veronis [20]; Phillips [18]). They are defined, however, as the eigenfunctions for an unfamiliar kind of eigenvalue problem, to which the ordinary completeness theory of Hilbert-Schmidt type is not applicable.

No strict argument therefore has been made, so far as the author knows, for the completeness of the whole set of Rossby normal modes, except for general conjectures based on physical intuition (Greenspan [8, 9]; Rhines and Bretherton [19]; Miller [15]). Recently an elementary proof was given of the completeness theorem for a

Received February 12, 1991.

1991 Mathematics Subject Classification. Primary 76C20, 49R20.

Current Address: Research Institute for Applied Mechanics, Kyushu University, Kasuga 816, Fukuoka, Japan.

(C)1993 Brown University 
special case of a rectangular basin (Masuda [13]). In that case, the eigenvalue problem was reduced to a one-dimensional problem by virtue of the simple rectangular form of the basin. Later the one-dimensional problem was generalized to a Sturm-LiouvilleRossby eigenfunction problem (Masuda [14]); it should be remarked that Mishoe [16] already studied a similar problem, though in a different way (see also Brauer [2]; DiPrima and G. J. Habetler [5]). Moreover, close relations turned out to exist between the Sturm-Liouville and the Sturm-Liouville-Rossby systems (Masuda [14]).

In the previous investigations, however, discussion was restricted to a one-dimensional problem or to the special case that can be reduced to a one-dimensional problem. This paper deals with an ocean with an arbitrary form of side boundary. The next section formulates the problem, and the third gives some preparations. In the fourth section we provide a proof of the completeness theorem for Rossby normal modes. Then, by virtue of the theorem, general solutions to the initial-value and the response problems are presented explicitly in terms of Rossby normal modes.

2. Formulation. We consider a closed ocean with a uniform depth $H$, where the (stable) density stratification and the side boundary may be arbitrary if they are appropriately smooth. The slow motion of the ocean at mid-latitudes is governed by the linear quasigeostrophic equation

$$
\frac{\partial}{\partial t}\left\{\nabla^{2} \Psi+\frac{\partial}{\partial z}\left(\frac{f_{0}^{2}}{N^{2}(z)} \frac{\partial \Psi}{\partial z}\right)\right\}+\beta \frac{\partial \Psi}{\partial x}=0
$$

in a bounded region $R \times(-H, 0)$, where $\Psi$ is the quasigeostrophic stream function, $(x, y, z)$ the (eastward, northward, vertical) coordinates, $\nabla$ the horizontal gradient operator, $f_{0}$ the characteristic value of the Coriolis parameter $f, \beta$ the meridional gradient of $f, N(z)$ the positive buoyancy frequency belonging to $C^{1}(-H, 0)$, and $R$ the two-dimensional area of the basin. The stream function must vanish at the side boundaries $\partial R \times(-H, 0)$ and satisfy the surface and bottom conditions:

$$
\left\{\begin{aligned}
\Psi & =0 & & \text { on } \partial R \times(-H, 0), \\
\frac{f_{0}^{2}}{N^{2}(0)} \frac{\partial^{2} \Psi}{\partial t \partial z} & =-\frac{\operatorname{curl} \sigma}{\rho_{0}} & & \text { at } z=0, \\
\frac{f_{0}^{2}}{N^{2}(-H)} \frac{\partial^{2} \Psi}{\partial t \partial z} & =0 & & \text { at } z=-H,
\end{aligned}\right.
$$

where $\sigma$ denotes the wind-stress on the sea surface and $\rho_{0}$ the characteristic density of sea water. The side boundary $\partial R$ is appropriately smooth; for example, it is sufficient that $\partial R$ be composed of finite smooth curves (Courant and Hilbert [3]; Wloka [21]). Also, the stream function must satisfy the initial condition

$$
\Psi=\Phi(x, y, z) \text { at } t=0 .
$$

For the above equations, the reader can refer to Gill [7] or Pedlosky [17]. 
In order to solve the problem defined by (1)-(3), we can expand the stream function in terms of the vertical mode $h_{k}(z)$ as

$$
\Psi(x, y, z, t)=\sum_{k=0}^{\infty} h_{k}(z) g_{k}(x, y, t),
$$

where $g_{k}$ indicates the Fourier coefficient for $h_{k}$. Here the vertical mode is defined by the differential equation

$$
\frac{d}{d z}\left\{\frac{f_{0}^{2}}{N^{2}(z)} \frac{d}{d z} h_{k}(z)\right\}+\nu_{k}^{2} h_{k}(z)=0,
$$

where $\nu_{k}^{2}$ denotes the eigenvalue that is nonnegative. The boundary conditions are

$$
\frac{d}{d z} h_{k}(z)=0 \text { at } z=0 \text { and }-H,
$$

and the normalization is made so that

$$
\int_{-H}^{0}\left|h_{k}(z)\right|^{2} d z=1
$$

Since (5) and (6) generate a Sturm-Liouville problem with the second kind of boundary condition, $\left\{h_{k}(z) \mid k=0\right.$ or $\left.k \in \mathbf{N}\right\}$ is a complete orthonormal set of the Hilbert space $H_{0}(-H, 0)$, where $\mathbf{N}$ is the set of positive integers. Hence the expression (4) is valid. The eigenfunction $h_{0}$ associated with the zero eigenvalue $\left(\nu_{k}=0\right)$ is called the barotropic mode, the first eigenfunction $h_{1}$ the first baroclinic mode, and so on.

Multiplying (1) by $h_{k}(z)$ and integrating over $z$ from $-H$ to 0 , we find that

$$
\frac{\partial}{\partial t}\left\{\nabla^{2} g_{k}-\nu_{k}^{2} g_{k}\right\}+\beta \frac{\partial g_{k}}{\partial x}=\frac{\operatorname{curl} \sigma}{\rho_{0} h_{k}(0)}
$$

where the boundary conditions (2) have been used. To solve (8), we have another eigenvalue problem defined by

$$
\left\{\begin{aligned}
\nabla^{2} \psi-\nu_{k}^{2} \psi+i \lambda \frac{\partial \psi}{\partial x} & =0 & & \text { in } R, \\
\psi & =0 & & \text { on } \partial R,
\end{aligned}\right.
$$

where $i$ denotes $\sqrt{-1}, \lambda$ the eigenvalue, and $\psi$ the eigenfunction. Note that the eigenvalue $\lambda$ is multiplied by a derivative of the eigenfunction rather than the eigenfunction itself. This difference makes it impossible to apply the usual completeness theory of Hilbert-Schmidt type. If the basin is rectangular with its two sides directed eastward, (9) can be reduced to a one-dimensional form, to which the theory of the Sturm-Liouville-Rossby equation is applied to assure the completeness of Rossby normal modes (Mishoe [16]; Masuda [13, 14]). For the other forms of basins such as a circular one, however, access to the completeness theorem by the methods used in the previous papers is hard. We therefore adopt a different approach; the proof obtained here, by the use of a variational principle, is of mathematical interest in itself (Courant and Hilbert [3, 4]). 
3. Eigenfunctions and their orthogonality. Let $D_{\infty}(R)$ be a space of $C^{\infty}$-class complex-valued functions that have compact supports in $R$. We define

$$
\begin{aligned}
(f, g) & =\iint_{R} f(x, y) g^{*}(x, y) d x d y, \\
(f, g)_{1} & =(f, g)+\left(\frac{\partial f}{\partial x}, \frac{\partial g}{\partial x}\right)+\left(\frac{\partial f}{\partial y}, \frac{\partial g}{\partial y}\right), \\
\langle f \mid g\rangle & =l^{2}(f, g)+\left(\frac{\partial f}{\partial x}, \frac{\partial g}{\partial x}\right)+\left(\frac{\partial f}{\partial y}, \frac{\partial g}{\partial y}\right),
\end{aligned}
$$

where an asterisk denotes the complex conjugate. The constant $l^{2}$ is zero for the barotropic mode and positive for the baroclinic modes. The corresponding norms are written as

$$
\begin{aligned}
\|f\| & =(f, f)^{1 / 2}, \\
\|f\|_{1} & =(f, f)_{1}^{1 / 2}, \\
\langle\langle f\rangle\rangle & =\langle f \mid f\rangle^{1 / 2} .
\end{aligned}
$$

Hilbert spaces $H_{0}(R)$ and $H_{1}(R)$ are defined as the completions of $D_{\infty}(R)$ with respect to the norms (13) and (14), respectively. As is well known, if $f$ is a function in $H_{1}(R)$, we have inequalities

$$
\begin{aligned}
& \|f\| \leq F \min \left\{\left\|\frac{\partial f}{\partial x}\right\|,\left\|\frac{\partial f}{\partial y}\right\|\right\} \leq F\|f\|_{1}, \\
& \|f\| \leq F\langle\langle f\rangle\rangle
\end{aligned}
$$

for a positive constant $F$.

We return to the eigenvalue problem (9). For the convenience of the following description, we rewrite (9) as

$$
\left\{\begin{aligned}
\mu\left(\nabla^{2} \psi-l^{2} \psi\right)+i \frac{\partial \psi}{\partial x} & =0 & & \text { in } R, \\
\psi & =0 & & \text { on } \partial R,
\end{aligned}\right.
$$

where we regard the inverse of the original eigenvalue $\lambda$ as the present eigenvalue $\mu$. Our concern is the completeness of the set of all the eigenfunctions of $(18)$ in $H_{0}(R)$ and $H_{1}(R)$.

Let $B$ be a bilinear functional on $H_{1}(R)$ defined by

$$
B(f, g)=-i \iint_{R} f \frac{\partial g^{*}}{\partial x} d x d y
$$

for $f$ and $g$ belonging to $H_{1}(R)$. It is easy to confirm the following relations:

$$
\begin{gathered}
\|B(f, g)\| \leq\|f\|\langle\langle g\rangle\rangle \leq F\langle\langle f\rangle\rangle\langle\langle g\rangle\rangle, \\
B(f, g)=B(g, f)^{*}, \\
B\left(f^{*}, g^{*}\right)=-B(g, f), \\
B(f+g, f+g)=B(f, f)+B(g, g)+2 \Re\{B(f, g)\}, \\
B(f+i g, f+i g)=B(f, f)+B(g, g)-2 \Im\{B(f, g)\},
\end{gathered}
$$


where $\Re$ and $\Im$ denote the real and the imaginary part, respectively.

The following two simple lemmas for the bilinear functional $B$ are frequently referred to later.

Lemma 1. If $f$ belongs to $H_{1}(R)$ and $\|f\|=0$, then $\|f\|_{1}$ and $\langle\langle f\rangle\rangle$ are zero.

Proof. For $\tilde{f}$ in $D_{\infty}(R)$, we have

$$
\begin{aligned}
\left\|\frac{\partial f}{\partial x}\right\|^{2} & \leq\left|\left(\frac{\partial f}{\partial x}, \frac{\partial \tilde{f}}{\partial x}\right)\right|+\left|\left(\frac{\partial f}{\partial x}, \frac{\partial(f-\tilde{f})}{\partial x}\right)\right| \\
& \leq\left|\left(f, \frac{\partial}{\partial x}\left(\frac{\partial \tilde{f}}{\partial x}\right)\right)\right|+\left\|\frac{\partial f}{\partial x}\right\| \cdot\left\|\frac{\partial(f-\tilde{f})}{\partial x}\right\| \\
& \leq\|f\| \cdot\left\|\frac{\partial^{2} \tilde{f}}{\partial x^{2}}\right\|+\langle\langle f\rangle\rangle \cdot\langle\langle f-\tilde{f}\rangle\rangle
\end{aligned}
$$

using the triangular and the Schwartz inequalities. Since $\|f\|=0$ and $f$ can be approximated arbitrarily well in $H_{1}(R)$ by a suitable $\tilde{f}$ in $D_{\infty}(R),\|\partial f / \partial x\|$ must be zero. Likewise $\|\partial f / \partial y\|$ vanishes, whence $\|f\|_{1}$ and $\langle\langle f\rangle\rangle$ are zero.

Lemma 2. If $f$ belongs to $H_{1}(R)$ and $B(f, g)$ is zero for any $g$ in $H_{1}(R)$, then $\langle\langle f\rangle\rangle=\|f\|_{1}=0$.

Proof. In the same way as in Lemma 1, we have

$$
\left\|\frac{\partial f}{\partial x}\right\|^{2} \leq\left|B\left(f, \frac{\partial \tilde{f}}{\partial x}\right)\right|+\langle\langle f\rangle\rangle \cdot\langle\langle f-\tilde{f}\rangle\rangle \rightarrow 0
$$

by choosing adequate $\tilde{f}$ in $D_{\infty}(R)$. Inequality (16) yields $\|f\|=0$. Since $f$ belongs to $H_{1}(R)$, Lemma 1 yields $\langle\langle f\rangle\rangle=\|f\|_{1}=0$.

Now we can consider the supremum $\mu_{1}$ of $B(f, f)$ for any $f$ with unit norm, since $B(f, f)$ is real and bounded from (21) and (20), respectively:

$$
\mu_{1}=\sup B(f, f)
$$

for $f$ in $H_{1}(R)$ and $\langle\langle f\rangle\rangle=1$.

First we show that $\mu_{1}$ is positive. If $\mu_{1}$ is negative, there is a function $f$ in $H_{1}(R)$ such that $\langle\langle f\rangle\rangle=1$, and $B(f, f) \leq \mu_{1}$. Eq. (22) shows that $B\left(f^{*}, f^{*}\right) \geq\left|\mu_{1}\right|>0$. Hence $\mu_{1}$ must be nonnegative. If $\mu_{1}$ equals zero, then $B(f, f)=0$ for any $f$ in $H_{1}(R)$. The identities (23) and (24) show that $B(f, g)$ is zero for any $f$ and $g$ in $H_{1}(R)$. Lemma 2 implies that any function in $H_{1}(R)$ is zero, which is incorrect. Thus, $\mu_{1}$ must be positive.

There exists a maximizing sequence $\left\{f_{n} \mid n \in \mathbf{N}\right\}$ of functions of unit norm in $H_{1}(R)$ for which the sequence $\left\{B\left(f_{n}, f_{n}\right)\right\}$ converges to $\mu_{1}$.

Proposition 1. Let $\left\{f_{n}\right\}$ be the above maximizing sequence. If $\left\{g_{n} \mid n \in \mathbf{N}\right\}$ is in $H_{1}(R)$ and $\left\langle\left\langle g_{n}\right\rangle\right\rangle \leq 1$, then

$$
\lim _{n \rightarrow \infty}\left|\mu_{1}\left\langle f_{n} \mid g_{n}\right\rangle-B\left(f_{n}, g_{n}\right)\right|=0 .
$$


Proof. From the definition of $\mu_{1}$, we have

$$
\begin{aligned}
0 \leq & \mu_{1}\left\langle\left\langle f_{n}+\varepsilon g_{n}\right\rangle\right\rangle^{2}-B\left(f_{n}+\varepsilon g_{n}, f_{n}+\varepsilon g_{n}\right) \\
= & {\left[\mu_{1}\left\langle\left\langle f_{n}\right\rangle\right\rangle^{2}-B\left(f_{n}, f_{n}\right)\right]+\varepsilon^{2}\left[\mu_{1}\left\langle\left\langle g_{n}\right\rangle\right\rangle^{2}-B\left(g_{n}, g_{n}\right)\right] } \\
& +2 \varepsilon \Re\left\{\mu_{1}\left\langle f_{n} \mid g_{n}\right\rangle-B\left(f_{n}, g_{n}\right)\right\}
\end{aligned}
$$

for any real $\varepsilon$. From the definition of $\mu_{1},(17)$, and (20) we have

$$
0 \leq \mu_{1}\left\langle\left\langle g_{n}\right\rangle\right\rangle^{2}-B\left(g_{n}, g_{n}\right) \leq \mu_{1}+F .
$$

For sufficiently large $n$, we have

$$
0 \leq \mu_{1}\left\langle\left\langle f_{n}\right\rangle\right\rangle^{2}-B\left(f_{n}, f_{n}\right) \leq \varepsilon^{2}\left(\mu_{1}+F\right),
$$

because $\left\{f_{n}\right\}$ is the maximizing sequence. Choosing the sign of $\varepsilon$ so that $\varepsilon \Re\left\{\mu_{1}\left\langle f_{n} \mid g_{n}\right\rangle-B\left(f_{n}, g_{n}\right)\right\}$ is negative, we obtain from (29)-(31) that

$$
\left|\Re\left\{\mu_{1}\left\langle f_{n} \mid g_{n}\right\rangle-B\left(f_{n}, g_{n}\right)\right\}\right| \leq|\varepsilon|\left(\mu_{1}+F\right) .
$$

Since $|\varepsilon|$ can be arbitrarily small for large $n$, the left-hand side of (32) tends to zero. Repeating the same argument with $g_{n}$ replaced by $-i g_{n}$, the imaginary part also vanishes as $n \rightarrow \infty$.

Proposition 2. For the maximizing sequence $\left\{f_{n}\right\}$ above,

$$
\lim _{m, n \rightarrow \infty}\left|\mu_{1}\left\langle\left\langle f_{m}-f_{n}\right\rangle\right\rangle^{2}-B\left(f_{m}-f_{n}, f_{m}-f_{n}\right)\right|=0 .
$$

Proof. As in (29), the following inequality holds:

$$
\begin{aligned}
& \left|\mu_{1}\left\langle\left\langle f_{n}-f_{m}\right\rangle\right\rangle^{2}-B\left(f_{n}-f_{m}, f_{n}-f_{m}\right)\right| \\
& \leq\left|\mu_{1}\left\langle\left\langle f_{n}\right\rangle\right\rangle^{2}-B\left(f_{n}, f_{n}\right)\right|+\left|\mu_{1}\left\langle\left\langle f_{m}\right\rangle\right\rangle^{2}-B\left(f_{m}, f_{m}\right)\right| \\
& \quad+2\left|\Re\left\{\mu_{1}\left\langle f_{n} \mid f_{m}\right\rangle-B\left(f_{m}, f_{n}\right)\right\}\right| .
\end{aligned}
$$

Since $f_{n}$ is the maximizing sequence, the first and second terms of the right-hand side decrease to zero. Proposition 1 shows that the third term becomes arbitrarily small as $m$ and $n$ increase to infinity, because the norm of $f_{n}$ is bounded by unity.

Proposition 3. There is a subsequence $\left\{g_{n}\right\}$ of the maximizing sequence $\left\{f_{n}\right\}$ such that $\left\{g_{n}\right\}$ converges to a function $g_{0}$ in $H_{1}(R)$.

Proof. Since $\left\langle\left\langle f_{n}\right\rangle\right\rangle=1$, Rellich's selection theorem (Courant and Hilbert $[3,4]$ ) assures the existence of a subsequence $g_{n}$ such that $\left\|g_{m}-g_{n}\right\|$ decreases to zero. For this subsequence, we obtain

$$
\lim _{m, n \rightarrow \infty}\left|B\left(g_{m}-g_{n}, g_{m}-g_{n}\right)\right|=0,
$$

since

$$
\begin{aligned}
\left|B\left(g_{m}-g_{n}, g_{m}-g_{n}\right)\right| & \leq\left|B\left(g_{m}-g_{n}, g_{m}\right)\right|+\left|B\left(g_{m}-g_{n}, g_{n}\right)\right| \\
& \leq\left\|g_{m}-g_{n}\right\|\left\{\left\langle\left\langle g_{m}\right\rangle\right\rangle+\left\langle\left\langle g_{n}\right\rangle\right\rangle\right\} \\
& \leq 2\left\|g_{m}-g_{n}\right\| .
\end{aligned}
$$


Since $\mu_{1}$ is positive, Proposition 2 and (35) give

$$
\begin{aligned}
\left\langle\left\langle g_{m}-g_{n}\right\rangle\right\rangle^{2} \leq & \frac{1}{\mu_{1}}\left|\mu_{1}\left\langle\left\langle g_{m}-g_{n}\right\rangle\right\rangle^{2}-B\left(g_{m}-g_{n}, g_{m}-g_{n}\right)\right| \\
& +\frac{1}{\mu_{1}}\left|B\left(g_{m}-g_{n}, g_{m}-g_{n}\right)\right| \rightarrow 0
\end{aligned}
$$

as $m$ and $n$ tend to infinity. From inequality (37) $\left\{g_{n}\right\}$ is a Cauchy sequence in the Hilbert space $H_{1}(R)$, which is complete. Hence, $g_{n}$ converges to a certain $g_{0}$ in $H_{1}(R)$ :

$$
\lim _{n \rightarrow \infty}\left\langle\left\langle g_{n}-g_{0}\right\rangle\right\rangle=\lim _{n \rightarrow \infty}\left\|g_{n}-g_{0}\right\|_{1}=0 .
$$

Proposition 4. The function $g_{0}(x, y)$ above is a weak solution of $(18)$ and $\tilde{g}_{0}(x, y)$ $=\exp \left\{-i x / 2 \mu_{1}\right\} g_{0}(x, y)$ is a weak solution of

$$
\left(\nabla^{2}-l^{2}\right) \tilde{g}_{0}+\frac{1}{4 \mu_{1}^{2}} \tilde{g}_{0}=0 .
$$

Proof. For any $f$ in $H_{1}(R)$, we have

$$
\mu_{1}\left\langle g_{0} \mid f\right\rangle-B\left(g_{0}, f\right)=0
$$

since

$$
\begin{aligned}
\left|\mu_{1}\left\langle g_{0} \mid f\right\rangle-B\left(g_{0}, f\right)\right| \leq & \left|\mu_{1}\left\langle g_{0}-g_{n} \mid f\right\rangle-B\left(g_{0}-g_{n}, f\right)\right| \\
& +\left|\mu_{1}\left\langle g_{n} \mid f\right\rangle-B\left(g_{n}, f\right)\right| \\
\leq & \left(\mu_{1}+F\right)\left\langle\left\langle g_{0}-g_{n}\right\rangle\right\rangle\langle\langle f\rangle\rangle+\left|\mu_{1}\left\langle g_{n} \mid f\right\rangle-B\left(g_{n}, f\right)\right| \rightarrow 0
\end{aligned}
$$

from (20), the Schwarz inequality, and Proposition 1. If $f$ belongs to $D_{\infty}(R)$, integrating (40) by parts, we obtain

$$
\iint_{R} g_{0}\left\{\mu_{1}\left(\nabla^{2}-l^{2}\right)-i \frac{\partial}{\partial x}\right\} f^{*} d x d y=0
$$

which shows $g_{0}$ to be a weak solution of (18). Replacing $f(x, y)$ by $\tilde{f}(x, y)=$ $\exp \left\{-i x / 2 \mu_{1}\right\} f(x, y)$ and $g_{0}(x, y)$ by $\tilde{g}_{0}(x, y)$ in (42), we find

$$
\iint_{R} \tilde{g}_{0}\left\{\left(\nabla^{2}-l^{2}\right)+\frac{1}{4 \mu_{1}^{2}}\right\} \tilde{f}^{*} d x d y=0 .
$$

That is, $\tilde{g}_{0}$ is a weak solution of (39), because $\tilde{f}$ can be any function in $D_{\infty}(R)$.

As is well known, weak solutions of elliptic equations can be regarded as genuine solutions, which really satisfy the boundary conditions required, in the sense that the norm of the difference between the weak solution and the genuine smooth solution is zero in $H_{0}(R)$ (Weyl's Lemma, see, e.g., Wloka [21]). Proposition 3 indicates that the weak solutions $g_{0}$ and $\tilde{g}_{0}$ obtained above belong to $H_{1}(R)$. Lemma 1 shows that the weak solutions equal the smooth genuine solutions as functions in $H_{1}(R)$. Therefore $\tilde{g}_{0}$ and $g_{0}$ are considered regular functions in $H_{1}(R)$. Thus we obtain the following theorem. 
THEOREM 1. The maximum value $\mu_{1}$ is attained by an eigenfunction $u_{1}(x, y)$ of the partial differential equation (18).

Obviously the minimum value of $B(f, f)$ subject to the condition $\langle\langle f\rangle\rangle=1$ is $\mu_{-1}=-\mu_{1}$, and $u_{-1}=u_{1}^{*}$ minimizes $B$. Also we have the orthgonality relations

$$
\left\langle u_{1} \mid u_{-1}\right\rangle=B\left(u_{1}, u_{-1}\right)=0 .
$$

From (21) and (40) it follows that if a function $f$ in $H_{1}(R)$ is orthogonal to $u_{1}$ (i.e., $\left.\left\langle f \mid u_{1}\right\rangle=0\right)$, then $B\left(f, u_{1}\right)=0$ and vice versa; the same is true for $u_{-1}$.

Next, we consider the similar maximizing problem with additional conditions

$$
\left\langle f \mid u_{1}\right\rangle=\left\langle f \mid u_{-1}\right\rangle=0 .
$$

Let $\mu_{2}$ be the supremum defined above:

$$
\mu_{2}=\sup B(f, f)
$$

for $f$ satisfying (45) and $\langle\langle f\rangle\rangle=1$. Then we can see that $\mu_{2}$ is positive as follows. The same argument as for $\mu_{1}$ shows that $\mu_{2}$ cannot be negative. Suppose that $\mu_{2}=0$. This implies that $B(f, f)=0$ for any function $f$ satisfying (45). Then, for any $f$ and $g$ subject to the above conditions, $B(f, g)=0$ by virtue of (23) and (24). Now, let $f$ be a function satisfying (45) and let $g$ be a function in $H_{1}(R)$. If we define

$$
\tilde{g}=g-\left\langle g \mid u_{1}\right\rangle u_{1}-\left\langle g \mid u_{-1}\right\rangle u_{-1},
$$

then we have

$$
\left\langle\tilde{g} \mid u_{1}\right\rangle=\left\langle\tilde{g} \mid u_{-1}\right\rangle=0 .
$$

Since $\tilde{g}$ satisfies (45) and $\mu_{2}=0$ from the assumption, it follows that

$$
B(f, \tilde{g})=0 .
$$

Hence we find

$$
\begin{aligned}
B(f, g) & =B(f, \tilde{g})+\left\langle g \mid u_{1}\right\rangle^{*} B\left(f, u_{1}\right)+\left\langle g \mid u_{-1}\right\rangle^{*} B\left(f, u_{-1}\right) \\
& =0 .
\end{aligned}
$$

Lemma 2 implies that $f$. equals zero. In other words, the functions orthogonal to $u_{1}$ and $u_{-1}$ must be zero; this implies $H_{1}(R)$ is spanned by two vectors $u_{1}$ and $u_{-1}$, which leads to a contradiction. Thus, $\mu_{2}$ must be positive.

There exists a maximizing sequence $\left\{f_{n}\right\}$ of unit norm subject to (45), for which $\left\{B\left(f_{n}, f_{n}\right)\right\}$ converges to $\mu_{2}$. Then Proposition 5 holds in the same way as Proposition 1 .

Proposition 5. Let $\left\{f_{n}\right\}$ be the above (second) maximizing sequence and $\left\{g_{n}\right\}$ be functions in $H_{1}(R)$ satisfying $\left\langle\left\langle g_{n}\right\rangle\right\rangle \leq 1$. Then,

$$
\lim _{n \rightarrow \infty}\left|\mu_{2}\left\langle f_{n} \mid g_{n}\right\rangle-B\left(f_{n}, g_{n}\right)\right|=0 .
$$

Proof. First, note that, if $g_{n}$ is bounded by unity and is orthogonal to $u_{1}$ and $u_{-1},(51)$ holds in just the same way as in the proof of Proposition 2 for $\mu_{1}$. Let 
$\tilde{g}_{n}$ be defined by (47), which is orthogonal to $u_{1}$ and $u_{-1}$. By the same calculation as for $(50)$, we have

$$
\mu_{2}\left\langle f_{n} \mid g_{n}\right\rangle-B\left(f_{n}, g_{n}\right)=\mu_{2}\left\langle f_{n} \mid \tilde{g}_{n}\right\rangle-B\left(f_{n}, \tilde{g}_{n}\right) .
$$

Since $1 \geq\left\langle\left\langle g_{n}\right\rangle\right\rangle \geq\left\langle\left\langle\tilde{g}_{n}\right\rangle\right\rangle$ (Bessel's inequality), (52) guarantees the proposition.

Following the same procedure as for $\mu_{1}$, we find that the second maximum $\mu_{2}$ is the second eigenvalue of (18) and that $\mu_{2}$ is attained by the associated eigenfunction $u_{2}$. It is easy to confirm

$$
\begin{aligned}
\left\langle u_{1} \mid u_{2}\right\rangle & =\left\langle u_{1} \mid u_{-2}\right\rangle=0, \\
B\left(u_{1}, u_{2}\right) & =B\left(u_{1}, u_{-2}\right)=0 .
\end{aligned}
$$

Thus we obtain the following result.

THEOREM 2. There are sequences of $\left\{\mu_{n}\right\}$ and $\left\{u_{n}\right\}$, where $\mu_{n}$ and $u_{n}$ are the eigenvalue and the associated eigenfunction, respectively, of the partial differential equation (18). The following orthogonality conditions hold:

$$
\begin{aligned}
\left\langle u_{m} \mid u_{n}\right\rangle & =\delta_{m, n}, \\
B\left(u_{m}, u_{n}\right) & =\mu_{m} \delta_{m, n},
\end{aligned}
$$

where $\delta_{m, n}$ is the Kronecker delta function.

Proposition 6. The dimension of the eigenspace associated with an eigenvalue is finite and $\mu_{n}$ decreases to zero as $n$ increases to infinity.

Proof. Suppose a positive eigenvalue has an infinite-dimensional eigenspace or $\mu_{n}$ does not tend to zero. Then, there is a positive lower bound $\alpha$ for the sequence $\left\{\mu_{n} \mid n \in \mathbf{N}\right\}$. Since $\left\langle\left\langle u_{n}\right\rangle\right\rangle=1$, Rellich's theorem guarantees the existence of a subsequence $\left\{v_{n}\right\}$ such that

$$
\lim _{m, n \rightarrow \infty}\left\|v_{m}-v_{n}\right\|=0 .
$$

Hence we have

$$
\begin{aligned}
\left|B\left(v_{m}-v_{n}, v_{m}-v_{n}\right)\right| & \leq\left\|v_{m}-v_{n}\right\|\left\{\left\langle\left\langle v_{m}\right\rangle\right\rangle+\left\langle\left\langle v_{n}\right\rangle\right\rangle\right\} \\
& =2\left\|v_{m}-v_{n}\right\| \rightarrow 0 .
\end{aligned}
$$

On the other hand, we have

$$
\begin{aligned}
B\left(v_{m}-v_{n}, v_{m}-v_{n}\right) & =B\left(v_{m}, v_{m}\right)+B\left(v_{n}, v_{n}\right) \\
& =\mu_{m}+\mu_{n} \geq 2 \alpha
\end{aligned}
$$

from (55) and (56). This contradiction between (58) and (59) proves the proposition.

4. Completeness theorems and general solutions. By virtue of the preceding preparations, we can now prove the main theorems.

THEOREM 3 (The completeness theorem for a specified vertical mode). The set of the eigenfunctions of (18) is a complete orthonormal set in $H_{1}(R)$; any function $f$ in $H_{1}(R)$ can be expanded as

$$
f(x, y)=\lim _{n \rightarrow \infty} f_{n}(x, y),
$$


where

$$
f_{n}(x, y)=\sum_{\substack{j=-n \\ j \neq 0}}^{n}\left\langle f \mid u_{j}\right\rangle u_{j}(x, y)
$$

in the sense that

$$
\lim _{n \rightarrow \infty}\left\|f-f_{n}\right\|=\lim _{n \rightarrow \infty}\left\langle\left\langle f-f_{n}\right\rangle\right\rangle=0 .
$$

That is, $\left\{f_{n}\right\}$ converges to $f$ strongly (norm convergence).

Proof. From the orthogonality conditions, we have

$$
\begin{gathered}
\left\langle\left\langle f-f_{n}\right\rangle\right\rangle^{2}=\langle\langle f\rangle\rangle^{2}-\sum_{\substack{j=-n \\
j \neq 0}}^{n}\left|\left\langle f \mid u_{j}\right\rangle\right|^{2} \geq 0, \\
B\left(f-f_{n}, f-f_{n}\right)=B(f, f)-\sum_{\substack{j=-n \\
j \neq 0}}^{n}\left|\left\langle f \mid u_{j}\right\rangle\right|^{2} \mu_{j} .
\end{gathered}
$$

Eq. (63) shows convergence of the following two series:

$$
\begin{gathered}
\sum_{\substack{j=-n \\
j \neq 0}}^{n}\left|\left\langle f \mid f_{j}\right\rangle\right|^{2}, \\
\sum_{\substack{j=-n \\
j \neq 0}}^{n}\left|\left\langle f \mid f_{j}\right\rangle\right|^{2}\left|\mu_{j}\right| \leq\left|\mu_{1}\right| \sum_{\substack{j=-n \\
j \neq 0}}^{n}\left|\left\langle f \mid f_{j}\right\rangle\right|^{2} .
\end{gathered}
$$

From the variational construction of the eigenfunctions $u_{j}$,

$$
0 \leq\left|B\left(f-f_{n}, f-f_{n}\right)\right| \leq \mu_{n+1}\left\langle\left\langle f-f_{n}\right\rangle\right\rangle \leq \mu_{n+1}\langle\langle f\rangle\rangle .
$$

Using (67), (23), and (24), we have, for any functions $f$ and $h$ in $H_{1}(R)$,

$$
\begin{aligned}
\left|B\left(f-f_{n}, h-h_{n}\right)\right| \leq & \left|\Re\left(B\left(f-f_{n}, h-h_{n}\right)\right)\right|+\left|\Im\left(B\left(f-f_{n}, h-h_{n}\right)\right)\right| \\
\leq & \left|B\left(f-f_{n}, f-f_{n}\right)\right|+\left|B\left(f-f_{n}, f-f_{n}\right)\right| \\
& +\frac{1}{2}\left|B\left(f-f_{n}+i\left(h-h_{n}\right), f-f_{n}+i\left(h-h_{n}\right)\right)\right| \\
& +\frac{1}{2}\left|B\left(f-f_{n}-i\left(h-h_{n}\right), f-f_{n}-i\left(h-h_{n}\right)\right)\right| \\
\leq & 2 \mu_{n+1}\left[\langle\langle f\rangle\rangle^{2}+\langle\langle h\rangle\rangle^{2}\right] .
\end{aligned}
$$

Since $\left(f-f_{n}\right)$ is orthogonal to $h_{n}, B\left(f-f_{n}, h-h_{n}\right)=B\left(f-f_{n}, h\right)$. Therefore we find that for any $h$ in $H_{1}(R)$

$$
\lim _{n \rightarrow \infty} B\left(f-f_{n}, h\right)=0,
$$

because $\mu_{n}$ decreases to zero as $n$ increases to infinity by virtue of Proposition 6 .

On the other hand, the absolute convergence of the series (65) implies

$$
\lim _{m, n \rightarrow \infty}\left\langle\left\langle\left(f-f_{n}\right)-\left(f-f_{m}\right)\right\rangle\right\rangle=\lim _{m, n \rightarrow \infty}\left\langle\left\langle f_{n}-f_{m}\right\rangle\right\rangle=0 .
$$


Since $\left(f-f_{n}\right)$ belongs to the complete space $H_{1}(R),\left(f-f_{n}\right)$ converges to a function $g$ in $H_{1}(R)$. Hence (69) is rewritten as

$$
B(g, h)=0,
$$

for any $h$ belonging to $H_{1}$. Lemma 2 is applied to show that $\|g\|=0$. Since $g$ is a function in $H_{1}(R)$, Lemma 1 shows that $\langle\langle g\rangle\rangle=\|g\|_{1}=0$. That is,

$$
g=\lim _{n \rightarrow \infty}\left(f-f_{n}\right)=0
$$

or

$$
f=\lim _{n \rightarrow \infty} f_{n} .
$$

The following is an almost trivial consequence of Theorem 3, (55)-(56), and Proposition 6. It corresponds to the converse of Theorem 1.

Proposition 7. Let $h$ be an eigenfunction of the differential equation (18) associated with an eigenvalue $\mu$. Then, $\mu$ belongs to $\left\{\mu_{n} \mid n \in \mathbf{Z}^{\prime}\right\}$ with $\mathbf{Z}^{\prime}$ being the set of the integers except for 0 , and $h$ is expressed as a finite sum of $u_{n}$ associated with the same eigenvalue $\mu$.

By combining Theorem 3 for the horizontal modes with the usual completeness theorem for the vertical modes (Sec. 2), we obtain the completeness theorem for Rossby normal modes as follows. Let $h_{k}(z)$ be the $k$ th vertical mode eigenfunction defined in Sec. 2 and let $u_{k, n}(x, y)$ be the $n$th eigenfunction defined in Sec. 3 associated with the vertical $k$ th mode. We define the $k, n$th Rossby normal mode $v_{k, n}(x, y, z)$ by

$$
v_{k, n}(x, y, z)=u_{k, n}(x, y) h_{k}(z) .
$$

Suppose $D_{\infty}(R \times \mathbf{R})$ and $D_{\infty}(R \times(-H, 0))$ are sets of $C^{\infty}$-class complex-valued functions that have compact supports in $R \times \mathbf{R}$ and $R \times(-H, 0)$, respectively, where $\mathbf{R}$ is the set of real numbers. On these spaces we define three inner products:

$$
\begin{gathered}
(f, g)_{D}=\int_{-H}^{0} \iint_{R}\left[f g^{*}\right] d x d y d z, \\
\langle f \mid g\rangle_{N}=\int_{-H}^{0} \iint_{R}\left[\nabla f \cdot \nabla g^{*}+\frac{f_{0}^{2}}{N^{2}(z)} \frac{\partial f}{\partial z} \frac{\partial g^{*}}{\partial z}\right] d x d y d z, \\
{[f, g]_{D}=(f, g)_{D}+\langle f \mid g\rangle_{N} ;}
\end{gathered}
$$

the corresponding norms being written as $\|\cdot\|_{D},\langle\langle\cdot\rangle\rangle_{N}$, and $[[\cdot]]_{D}$, respectively. The suffix $N$ of (76) indicates the inner product relevant for normal modes; the square of the norm $\langle\langle f\rangle\rangle_{N}^{2}$ is the total energy (kinetic plus available potential) of the motion " $f$ ". The Hilbert space $D$ we use here is defined as the completion of $D_{\infty}(R \times \mathbf{R})$ with respect to the norm $[[\cdot]]_{D}$. The two norms $\langle\langle\cdot\rangle\rangle_{N}$ and $[[\cdot]]_{D}$ are equivalent as a distance, because inequalities like (16) and (17) are valid in $D$ as well.

THEOREM 4 (The completeness theorem and expansion theorem for Rossby normal modes). The Rossby normal modes (74) make a complete orthonormal set in $D$ 
with respect to $\langle\cdot \mid \cdot\rangle_{N}$. Any function $f$ in $D$ can be expanded as

$$
f=\sum_{k=0}^{\infty} \sum_{\substack{n=-\infty \\ n \neq 0}}^{\infty}\left\langle f \mid v_{k, n}\right\rangle_{N} v_{k, n}(x, y, z),
$$

where the right-hand side converges strongly.

Proof. Suppose that $f$ belongs to $D$ and that $\left\langle f \mid v_{k, n}\right\rangle_{N}=0$ for any $k$ and $n$. As is well known, it suffices to show $\langle\langle f\rangle\rangle_{N}=[[f]]_{D}=0$ for such a function $f$. The orthogonality of $f$ with $v_{k, n}$ is rewritten as

$$
\int_{-H}^{0} \iint_{R} f(x, y, z) \frac{\partial u_{k, n}^{*}(x, y)}{\partial x} h_{k}(z) d x d y d z=0
$$

since $v_{k, n}(x, y, z)$ is a Rossby normal mode (eigenfunction). The integrand of (79) is absolutely integrable, so that Fubini's theorem can be applied to show that

$$
g_{k}(x, y)=\int_{-H}^{0} f(x, y, z) h_{k}(z) d z
$$

is significant for a.e. $(x, y)$ in $R$, and (79) becomes

$$
\iint_{R} g_{k}(x, y) \frac{\partial u_{k, n}^{*}}{\partial x} d z=0 .
$$

Again from Fubini's theorem and the Schwarz inequality, we obtain

$$
\begin{aligned}
& \iint_{R}\left|g_{k}(x, y)\right|^{2} d x d y \\
& \quad \leq \iint_{R}\left\{\int_{-H}^{0}|f(x, y, z)|^{2} d z \times \int_{-H}^{0}\left|h_{k}(z)\right|^{2} d z\right\} d x d y
\end{aligned}
$$

which shows $g_{k}(x, y)$ is an element of $H_{0}(R)=L_{2}(R)$. In the same way, we can show that

$$
\begin{aligned}
& \frac{\partial g_{k}(x, y)}{\partial x}=\int_{-H}^{0} \frac{\partial f(x, y, z)}{\partial x} h_{k}(z) d z, \\
& \frac{\partial g_{k}(x, y)}{\partial y}=\int_{-H}^{0} \frac{\partial f(x, y, z)}{\partial y} h_{k}(z) d z
\end{aligned}
$$

are functions in $H_{0}(R)$, where $\partial f / \partial x$ and $\partial f / \partial y$ denote weak derivatives of $f$. It remains to confirm that (83) and (84) express the weak first derivatives of $g_{k}(x, y)$. Let $s(x, y)$ be a function in $D_{\infty}(R)$. It is approximated by $s(x, y) \cdot t(z)$ in $D_{\infty}(R \times(-H, 0))$ with $t(z)$ an appropriate smooth function in $R \times(-H, 0)$ such that $\|s \cdot t-s\|_{D} \rightarrow 0$. Since $s t$ belongs to $D_{\infty}(R \times(-H, 0))$ and $f$ has weak first derivatives in $R \times(-H, 0)$, we have

$$
\begin{aligned}
\int_{-H}^{0} & \iint_{R} \frac{\partial f(x, y, z)}{\partial x} h_{k}(z) s(x, y) t(z) d x d y d z \\
& =-\int_{-H}^{0} \iint_{R} f(x, y, z) h_{k}(z) \frac{\partial s(x, y)}{\partial x} t(z) d x d y d z .
\end{aligned}
$$


As the limit of (85), (83) represents the weak derivative of $g_{k}$ with respect to $x$. The same is true for (84).

Since $f$ belongs to $D$, there is a sequence $\left\{f_{m} \mid m \in \mathbf{N}\right\}$ of elements in $D_{\infty}(R \times(-H, 0))$ that converges to $f$ in $D$. Let us define

$$
g_{k, m}(x, y)=\int_{-H}^{0} f_{m}(x, y, z) h_{k}(z) d z
$$

which is an element of $D_{\infty}(R)$. Obviously we have

$$
\lim _{m \rightarrow \infty}\left\|g_{k, m}(x, y)-g_{k}(x, y)\right\|_{1}=0,
$$

which implies that $g_{k}(x, y)$ belongs to $H_{1}(R)$.

From the use of Lemma 2 and Theorem 3 for each vertical mode, it follows from (81) that $\left\|g_{k}\right\|_{1}=\left\|g_{k}\right\|=0$. Hence $g_{k}(x, y)$ is 0 for a.e. $(x, y)$ in $R$. Since (80) holds for any $k$, the completeness theorem for the vertical modes assures that, for a.e. $(x, y)$ in $R, f(x, y, z)=0$ at a.e. $z$ in $(-H, 0) ; f(x, y, z)=0$ for a.e. $(x, y, z)$ in $R \times(-H, 0)$ and consequently $\|f\|_{D}=0$.

With respect to $[[\cdot]]_{D},\left\{f_{m} \in D_{\infty}(R \times \mathbf{R}) \mid m \in \mathbf{N}\right\}$ converges to $f$. For each $m$, there are sequences $\left\{f_{l, m, x} \mid l \in \mathbf{N}\right\},\left\{f_{l, m, y} \mid l \in \mathbf{N}\right\}$ and $\left\{f_{l, m, z} \mid l \in \mathbf{N}\right\}$ in $D_{\infty}(R \times(-H, 0))$, such that

$$
\begin{aligned}
& \lim _{l \rightarrow \infty}\left\|\frac{\partial f_{m}}{\partial x}-f_{l, m, x}\right\|_{D}=0, \\
& \lim _{l \rightarrow \infty}\left\|\frac{\partial f_{m}}{\partial y}-f_{l, m, y}\right\|_{D}=0, \\
& \lim _{l \rightarrow \infty}\left\|\frac{\partial f_{m}}{\partial z}-f_{l, m, z}\right\|_{D}=0 .
\end{aligned}
$$

Then, an argument similar to the one in Lemma 1 shows that $\langle\langle f\rangle\rangle_{N}=[[f]]_{D}=0$.

Now we write down the general solution to the problem given by (1)-(3). The corresponding characteristic frequency of the basin becomes

$$
\omega_{k, n}=\beta \mu_{k, n} .
$$

According to the usual calculation (Masuda [13]), we obtain the following general solution to the initial-value problem and the response problem (1)-(3):

$$
\begin{aligned}
\Psi(x, y, z, t)= & \sum_{k=0}^{\infty} \sum_{\substack{n=-\infty \\
n \neq 0}}^{\infty} v_{k, n}(x, y, z) \\
& \times\left[\left\langle\Phi \mid u_{k, n}\right\rangle_{N} e^{-i \omega_{k, n} t}+\int_{0}^{t}\left(-\frac{\operatorname{curl} \sigma}{\rho_{0} h_{k}(0)}, u_{k, n}\right) e^{-i \omega_{k, n}(t-\tau)} d \tau\right] .
\end{aligned}
$$

In particular, the response of the basin to an instantaneous torque located at $(\xi, \eta)$ at time $t=\tau$ is found by setting $\Phi=0$ and $\operatorname{curl} \sigma / \rho_{0}=\delta(x-\xi) \delta(y-\eta) \delta(t-\tau)$, 
where $\delta$ denotes the delta function. That is, Green's function formally becomes

$$
\begin{aligned}
G(x, y, z, t ; \xi, \eta, \tau) \\
\quad=-\sum_{k=0}^{\infty} \frac{h_{k}(z)}{h_{k}(0)} \sum_{\substack{n=-\infty \\
n \neq 0}}^{\infty} u_{k, n}(x, y) u_{k, n}(\xi, \eta) e^{-\omega_{k, n}(t-\tau)} .
\end{aligned}
$$

5. Discussion. In this paper, a proof is obtained of the completeness of Rossby normal modes in a basin with an arbitrary form of side boundary. This investigation, however, is only a beginning of the study of completeness of characteristic oscillations appearing in geophysical fluid dynamics (see LeBlond and Mysak [10], for a variety of wave motions found in the atmosphere and the ocean). In fact, the present results apply merely to quasigeostrophic dynamics in basins with a uniform depth. When the bottom is uneven and the ocean is stratified, the problem becomes essentially three-dimensional. If ageostrophy is taken into consideration, the problem becomes that of vector-valued eigenfunctions (Masuda [14]). When the ocean has a boundary of zero depth or an infinite domain, a careful analysis of singularities is required. These are subjects to be studied in the future.

Acknowledgment. I would like to thank Professors K. Kajiura and H. Mitsuyasu for their encouragement.

\section{REFERENCES}

[1] D. L. T. Anderson and A. E. Gill, Spin-up of a stratified ocean, with application to upwelling, Deep-Sea Research 22, 583-596 (1975)

[2] F. Brauer, Spectral theory for the differential equation $L u=\lambda M u$, Canad. J. Math. 10, 431-446 (1958)

[3] R. Courant and D. Hilbert, Methoden der Mathematischen Physik, Vol. 2, Verlag von Julius Springer, Berlin, 1937

[4] __. Methods of Mathematical Physics, Interscience, New York, Vol. 1, 1953, Vol. 2, 1962

[5] R. C. DiPrima and G. J. Habetler, A completeness theory for nonselfadjoint eigenvalue problems in hydrodynamic stability, Arch. Rational Mech. Anal. 34, 218-227 (1969)

[6] E. Firing and R. C. Beardsley, The behavior of a barotropic eddy on a beta-plane, J. Phys. Oceanogr. 6, 57-65 (1976)

[7] A. E. Gill, Atmosphere-Ocean Dynamics, Academic Press, San Diego, CA, 1982

[8] H. P. Greenspan, On the transient motion of a contained rotating fluid, J. Fluid Mech. 20, 673-696 (1964)

[9] __ On the general theory of contained rotating fluid motions, J. Fluid Mech. 22, 449-462 (1965)

[10] P. H. LeBlond and L. A. Mysak, Waves in the Ocean, Elsevier, Amsterdsam, 1978

[11] M. J. Lighthill, Dynamic response of the Indian Ocean to onset of the southwest monsoon, Philos. Trans. Roy. Soc. London 265, 45-92 (1969)

[12] M. S. Longuet-Higgins, Planetary waves on a rotating sphere, Proc. Roy. Soc. London A279, 446$473(1964)$

[13] A. Masuda, $A$ proof and applications of the expansion theorem for the Rossby normal modes in a closed rectangular basin, J. Oceanogr. Soc. Japan 43, 237-243 (1987)

[14] _ On the completeness and the expansion theorem for eigenfunctions of the Sturm-LiouvilleRossby type, Quart. Appl. Math. 47, 435-445 (1989)

[15] A. J. Millar, Nondivergent planetary oscillations in midlatitude ocean basins with continental shelves, J. Phys. Oceanogr. 16, 1914-1928 (1986)

[16] L. I. Mishoe, Fourier series and eigenfunction expansions associated with a nonselfadjoint differential equation, Quart. Appl. Math. 20, 175-181 (1962)

[17] J. Pedlosky, Geophysical Fluid Dynamics, 2nd ed., Springer-Verlag, Berlin and New York, 1987 
[18] N. Phillips, Large-scale eddy motion in the western Atlantic, J. Geophys. Res. 71, 3883-3891 (1966)

[19] P. B. Rhines and F. Bretherton, Topographic Rossby waves in a rough-bottomed ocean, J. Fluid Mech. 61, 583-604 (1973)

[20] G. Veronis, Rossby waves with bottom topography, J. Marine Res. 24, 338-349 (1966)

[21] J. Wloka, Partial Differential Equations, Cambridge Univ. Press, London and New York, 1987 